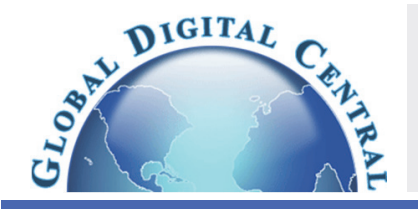

Frontiers in Heat and Mass Transfer

\title{
NUMERICAL STUDY OF THE GASKET THERMAL CONDUCTIVITY EFFECT ON THE THERMAL CONTACT RESISTANCE BETWEEN TWO SOLIDS IN CONTACT
}

\author{
Rachid Chadouli $^{\mathrm{a} \mathrm{b}^{*}}$, Frédéric Lebon $^{\mathrm{a}}$, Iulian Rosu $^{\mathrm{a}}$, Mohammed Makhlouf $^{\mathrm{b}}$ \\ a Aix Marseille University, CNRS, Centrale Marseille, LMA, UPR 7051, Marseille, France \\ ${ }^{b}$ Mechanical Engineering Department, Faculty of Technology, Djillali Liabes University Sidi Bel Abbes 22000 Algeria
}

\begin{abstract}
In this paper, a numerical model investigating the impact of gasket thermal quality on the reduction in the thermal contact resistance (TCR) between two solids is presented and validated analytically. The model proposed is a 2D steady state model. The thermal conductivity of the solid materials ranges from 20 to $390 \mathrm{~W} / \mathrm{m} \cdot \mathrm{K}$, and the gasket thermal conductivity ranges from $0.16 \mathrm{~W} / \mathrm{m} \cdot \mathrm{K}$ (TC of rubber) to and $5 \mathrm{~W} / \mathrm{m} \cdot \mathrm{K}$ (TC of thermal paste). As expected, the results obtained clearly confirm that the gasket significantly improves the heat transfer between two solids in contact, and in particular that the TCR is decreased with increasing gasket thermal conductivity. The numerical results are validated by an analytical model which shows that the gasket has a crucial effect on the heat transfer enhancement between the solids in contact.
\end{abstract}

Keywords: contact, asperities, thermal contact resistance, gasket, thermal conductivity.

\section{INTRODUCTION}

It is well known that the contact between two solids is not perfect particularly because of the irregularities of contact surfaces due to the presence of asperities. For this reason, the heat transfer between the two solids occurs mainly through the contact points, and the assumption of thermal contact resistance is present because of the unevenness of interfaces in contact. This thermal contact resistance (TCR) is a barrier to heat flow resulting in dramatic temperature drop. This phenomenon results from the disturbance of the heat flux at the contact. The (TCR) is influenced by the size of surface roughness "Ra" and by the interstitial medium, which is generally a bad conductor. Therefore improving heat transfer between two contact materials means reducing the TCR, which requires installing a gasket between the two solids in contact. This gasket can be a thermal paste, or rubber.

The thermal contact resistance is considered as a key parameter in mechanical contacts. It plays an important role in the heat transfer between solids in contact. Heat transfer enhancement requires to study and to model the gasket effect on the decrease in TCR. Given the importance of the thermal contact resistance, a lot of numerical and experimental research has been carried out. Voller et al. (2007) analyzed the (TCR) across a bolted joint brake disc-pad. They investigated two methods to reduce the TCR: the application of thermal paste between solids in contact and the use of a thin aluminum gasket at the interface. The results obtained show that these techniques reduce the TCR by more than $80 \%$. O'Callaghan et al. (1988) developed a computer-based mathematical model to predict the optimal thickness for the interfacial insert between two solids, which will minimize the thermal resistance of the contact assembly. The model was used to predict the behavior of a joint formed between the flat surfaces of two solids. The results obtained were validated experimentally and a good agreement was achieved (error rate less than $15 \%$ ).
Zhang et al. (2010) developed an experimental device for measuring interfacial contact resistance at the interface between two materials in contact separated by a lubricant. They evaluated the impact of the contact pressure, the interface temperature and the thickness of the lubricant on the interfacial contact resistance. The results obtained show that the interfacial contact resistance is, on the one hand, strongly influenced by these parameters and, on the other hand, greatly reduced when a lubricant gasket is used. Loulou et al. (1999) developed an experimental study to simulate the real contact conditions during glass solidification and built a numerical procedure to estimate the thermal parameters (left and right temperatures, heat flux at interface and contact resistance) characterizing the thermal phenomena that influence the contact interface. They constructed a mathematical model to estimate the thermal contact resistance between glass and mould during the glass cooling process. The results obtained show that when the coupled conduction-radiation effect is taken into account, the parameter estimation is better.

Degiovanni et al. (1998) performed a three-dimensional analytical study to estimate the TCR. They proposed a microscopic modeling of the cylinder-cylinder contact in the steady state. They represented the TCR using a three resistance network, and identified the constriction resistance. The results obtained show that the constriction resistance for a cylinder-cylinder contact may be assimilated to that of a plane-plane contact. Cames-Pintaux et al. (1980) proposed an equivalent thermal model for imperfect contacts. They assumed that the contact between the two materials is equivalent to three walls in series, the medium is also composed of two parallel walls that one of them have the same thermal conductivity as the two outer walls and the other represents the interstitial medium. The numerical study is based on the comparison of changes in temperature between the two materials using the pairs: concrete-air, brass-air and concrete-polystyrene. The authors confirmed that the contact can be modeled as parallel strips.

Dongmi et al. (2012) measured the thermal contact resistance between two solids, within a temperature range of 70-290 K and a pressure range of 0.2-0.7 $\mathrm{MPa}$ using the Laser Photo Thermal method,

\footnotetext{
${ }^{*}$ Corresponding author.Email: chadouli@lma.cnrs-mrs.fr
} 
which is a transient and non-contact method. Results show that the TCR decreased with the increasing temperature, and that this change is significant when the temperature is lower than $150 \mathrm{~K}$. The contact pressure can enlarge the real contact area, which can cause the TCR decrease, but with a little effect compared to the effect of the interface temperature.

Dureja et al. (2015) developed an experimental setup and procedures to determine the thermal contact conductance of disc shaped flat specimens. The results obtained indicate that the thermal contact conductance values are linear functions of the contact pressure in the range of 1-10 MPa. Ruifeng et al. (2016) presented an experimental study to investigate the effects of interface temperature in the region of 360$640^{\circ} \mathrm{C}$ on the thermal contact conductance (TCC) (specimen surface roughness ranged from $0.25 \mu \mathrm{m}$ to $2 \mu \mathrm{m}$, and contact pressure between 2.39 and $15.17 \mathrm{MPa}$ ). Results indicated that TCC presents a power-law relationship with these parameters.

Belghith et al. (2010) investigated the contact between a rough deformable surfaces and a smooth rigid plan and developed a microscopic, deterministic and analytical contact model taking into account the properties of engineering surfaces. Geometrical characteristics of rough surfaces are deduced using the standard procedure for roughness and waviness parameters. As the microscopic model often enables the real structure with complex geometry to be simulated, the authors developed a homogenization technique and the interface of the equivalent model was governed by the microscopic model results.

Belghith et al. (2013) developed a thermo mechanical model of a contact between two rough surfaces. They studied a rough-smooth surface contact by applying pressure to reduce the interstitial space and thereby reduce the TCR. The results obtained show that the TCR and the interstitial space between the two contacting surfaces are decreased by increasing pressure. Quigyun et al. (2015) performed an experimental study in order to show the influencing factors of thermal contact conductance (TCC) between TC4 and 30CrMnSi interfaces which are the main building materials used in aviation engines. Results show that the temperature in the region of $200^{\circ} \mathrm{C}-350^{\circ} \mathrm{C}$ has little effect on TCC. The TCC reaches its peaks value at $120 \mathrm{MPa}$. Under the same condition, rough surfaces exhibit higher TCC values than smooth surfaces.

Navni et al. (2016) used a numerical method to extracting the TCC of metal-metal interfaces. A scale-resolved direct numerical simulation of conjugate heat transfer across the interface was developed. Results show that the conductance is strongly related to the number of contact points and hollow in the contact form. Based on this consideration, the authors proposed a simplified model in which the thermal conductance is assumed to be a function of the number of contacts only. Benjamin et al. (2016) measured the TCC between aluminum surfaces in the pressure range from $0.172 \mathrm{MPa}$ to $2.76 \mathrm{MPa}$. The results obtained show that the TCC at $2.76 \mathrm{MPa}$ is greater than the TCC at $0.172 \mathrm{MPa}$.

Zhi et al. (2013) developed an experimental setup based on the steady state heat flux method to measure the thermal contact conductance at the interfaces of Hastelloy C-276/ Hastelloy C-276 and Hastelloy C$276 /$ ss 302 based on the steady state heat flux method. Results show that the TCC between this pairs increases with the increase in average interfacial temperature and a highest values are found for temperatures of about $346^{\circ} \mathrm{C}$ and the lowest about $214^{\circ} \mathrm{C}$.

Considering these studies, and given that most of works carried out in this field are based on the gasket thickness impact on the TCR without taking into account of its thermal conductivity effect, the objective of the present work was the study of the gasket thermal conductivity effect on the the TCR in order to improve heat transfer between solids contacts. The commercial software Abaqus (6.14) was utilized to compute numerically the thermal contact resistance between rough surfaces as a function of the gasket thermal conductivity using the Finite Element Method.

The paper is decomposed as follow: Section 2 is devoted to the methodology. An analytical model is introduced, then a numerical procedure based on the Finite Element Method is proposed. In Section 3, numerical results are presented: first, the effect of the gasket on the thermal contact resistance is studied, then the rate of decrease in the thermal contact resistance is analyzed. The paper ends with conclusions and perspectives.

\section{METHODOLOGY}

\subsection{Analytical Model}

In this paper, the contact between two rough solids is studied. For the sake of simplicity, it is considered that the asperities are square shaped and that the contact is assimilated to the contact of three solids in series (see Fig.1); the middle one is composed by two parallel solids, one of them having the same thermal conductivity $\mathrm{k}_{1}$ than the two outer solids and representing the contact through the asperities. The other inner solid is an interstitial medium with thermal conductivity $\mathrm{k}_{2}$ which represents the gasket (Cames-Pintaux et al., 1980). In the following, only two dimensional solids are considered.

In the following the width is $\mathrm{D}=0.02 \mathrm{~m}$. As mentioned on "Fig. 1 ," the height of the outer solids is $0.08 \mathrm{~m}$ and the gasket thickness is $\left(\mathrm{Ra}^{*} 2\right)$. where $\mathrm{Ra}$ is taken to denote the surfaces roughness.

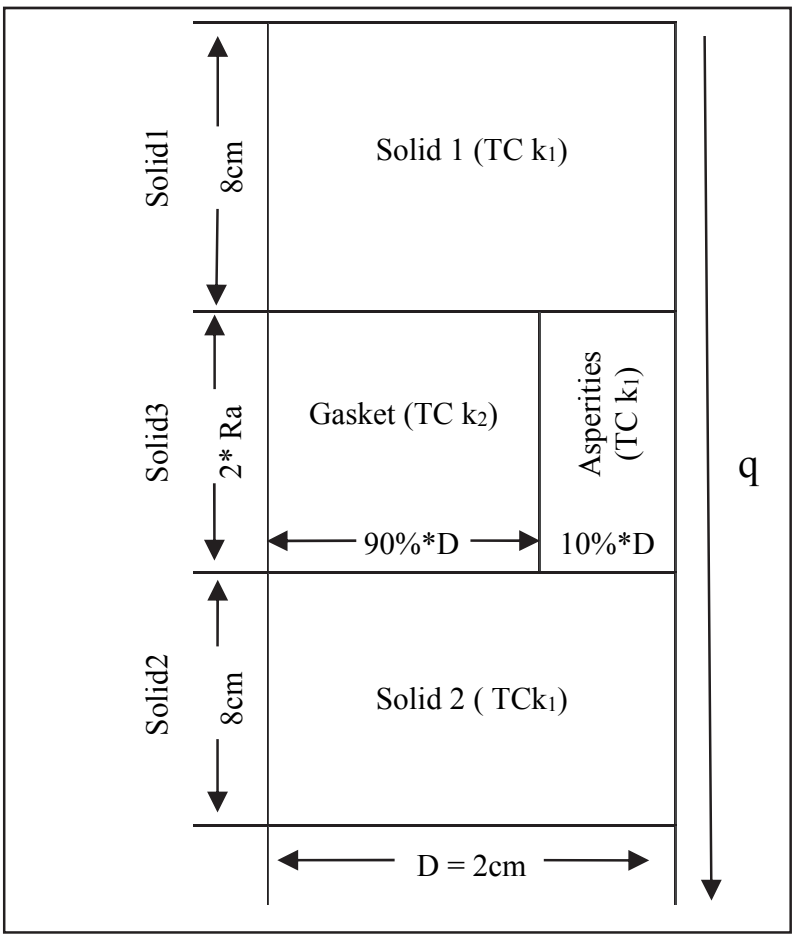

Fig.1 Schematic equivalent walls

The actual contact between the two solids should not exceeds $10 \%$ of nominal contact area for smooth surfaces (Madhusudana, 1996). For this reason, it is assumed that the gasket surface represents $90 \%$ of the total width $\mathrm{D}$.

Let $\mathrm{T}$ denote the temperature field and $\mathrm{Z}$ to denote the longitudinal coordinate. According to the Fourier law of conduction, the heat flux $\mathrm{q}$ in the transition area can be defined by the sum of two heat fluxes, one through the number $\mathrm{N}$ of asperities, which is defined by $\mathrm{q}_{1}$ and the other through the interstitial medium, which is defined by $\mathrm{q}_{2}$.

$q=q_{1}+q_{2}$ 


$$
q=-k_{1} S_{1} \frac{d T}{d z}-k_{2} S_{2} \frac{d T}{d z}
$$

$\mathrm{S}_{1}$ and $\mathrm{S}_{2}$ are both surfaces which emit the $\mathrm{q}_{1}$ and $\mathrm{q}_{2}$ fluxes, respectively defined by:

$$
\begin{aligned}
& S_{1}=R a \cdot N \\
& S_{2}=D-R a \cdot N
\end{aligned}
$$
equation:

The thermal contact resistance TCR was defined by the following

$$
T C R=\frac{\Delta T}{q}
$$

Where $\Delta \mathrm{T}$ is taken to denote the temperature jump between the two outer solids, defined by:

$$
\Delta T=T_{c h}-T_{f}
$$

From the resolution of Eq. (2) and the definition of the thermal contact resistance Eq. (5), the analytical model of the thermal contact resistance used in the study is given by:

$$
T C R=\frac{2 R a}{k_{1} S_{1}+k_{2} S_{2}}
$$

\subsection{Numerical Model}

In this work, numerical simulations are based on a steady state 2D model using the Finite Element Method and obtained by ABAQUS (6.14). The mesh convergence is studied in order to obtain a good compromise between computational cost and convergence. The mesh used in this simulation is composed of standard elements with linear interpolation and heat transfer type. The mesh is composed of quadrilateral elements (DC2D4) and Computation is performed by the "minimize the mesh transition" algorithm using the structured meshing technique, the number of elements being $n=[(9950+2500) \times 2]+(25 \times 99)=27375$ elements .

It is recalled that the two outer solids in contact each have a height of $8 \mathrm{~cm}$ and a width of $2 \mathrm{~cm}$. The rough surfaces of two solids in contact are modeled by square asperities surfaces with the same size $\mathrm{Ra}=20 \mu \mathrm{m}$ and same distance between them, the actual contact rate is assumed to be $10 \%$ of the total area A. The thermal conductivity of the materials in contact ranges from 20 to $390 \mathrm{w} / \mathrm{m} \cdot \mathrm{K}$. The interstitial medium is assimilated as a material with thermal conductivity $\mathrm{k}_{2}$ which ranges between 0.16 and $5 \mathrm{w} / \mathrm{m} \cdot \mathrm{K}$.

In order to evaluate numerically the temperatures $\left(\mathrm{T}_{\mathrm{ch}}\right)$ and $\left(\mathrm{T}_{\mathrm{f}}\right)$ near the contact interface (see Fig.2 and Fig.3), to compute the heat flow q and to determine the TCR, the outer surface of the upper material is heated at $538 \mathrm{~K}$ and the lower surface of the bottom material at $338^{\circ} \mathrm{K}$, we consider that the laterals surfaces are insulated. 2016):

The thermal contact resistance is defined as Eq. (8) (Ruifeng et al.,

$T C R=\frac{T_{c h}-T_{f}}{q}$

$\mathrm{q}$ being the average heat flow between the two materials in contact

$$
\begin{aligned}
& q=\frac{q_{c h}+q_{f}}{2} \\
& q=\frac{1}{2}\left[-k_{1} \cdot S \cdot\left(\frac{d T}{d z}\right)_{c h}-k_{1} \cdot S \cdot\left(\frac{d T}{d z}\right)_{f}\right]
\end{aligned}
$$

$$
\begin{aligned}
& q_{c h}=\frac{k_{1} S}{z_{c h}}\left(T_{a}-T_{c h}\right) \\
& q_{f}=\frac{k_{1} S}{z_{b}-z_{f}}\left(T_{f}-T_{b}\right)
\end{aligned}
$$

$\mathrm{S}$ is taken to denote the surface which emits the heat flow $\mathrm{q}$

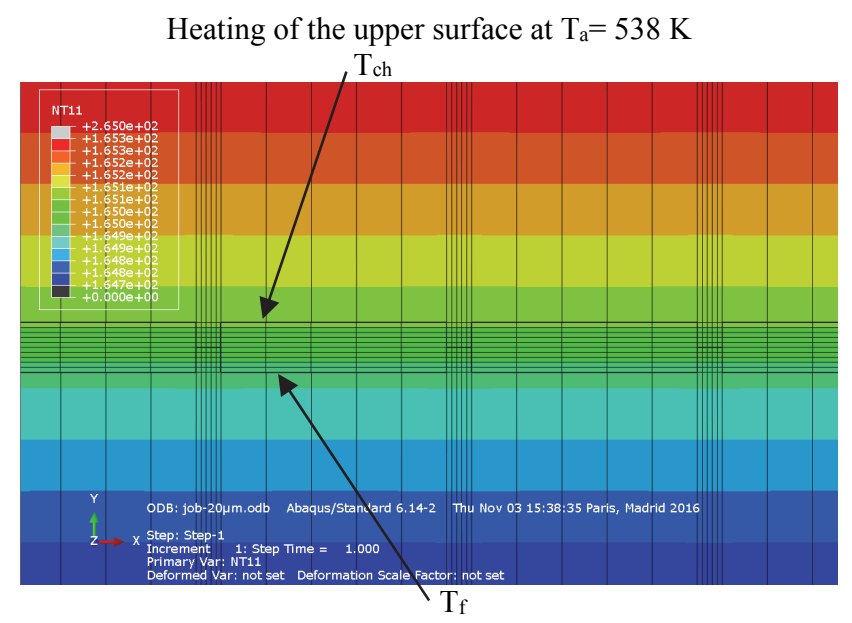

Heating of the lower surface at $\mathrm{T}_{\mathrm{b}}=338 \mathrm{~K}$

Fig.2 Geometrical model of refined mesh around the contact interface.

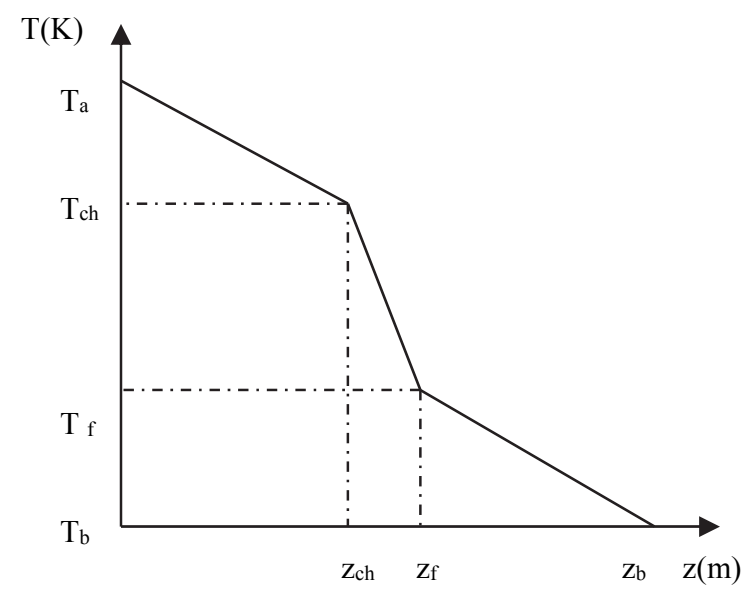

Fig. 3 Temperature profile of an imperfect contact

\section{RESULTS AND DISCUSSION}

\subsection{Effect of the gasket on the thermal contact resistance}

In order to study the impact of the type of gasket on the thermal contact resistance, the thermal contact resistance has been computed as a function of the gasket thermal conductivity. The computations were done on three pairs of materials with thermal conductivity $20 \mathrm{w} / \mathrm{m} \cdot \mathrm{K}, 220$ $\mathrm{w} / \mathrm{m} \cdot \mathrm{K}$ and $390 \mathrm{w} / \mathrm{m} \cdot \mathrm{K}$ respectively and validated by the analytical model. 

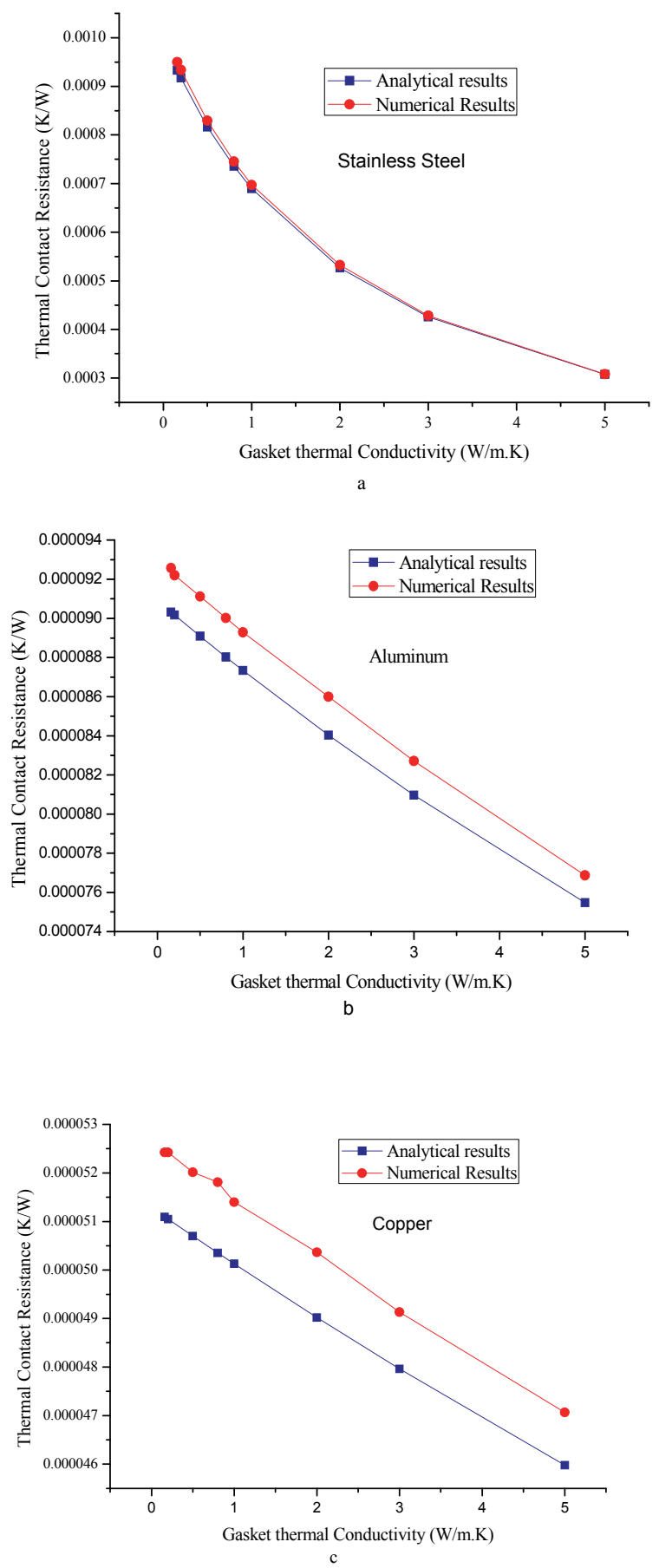

Fig. 4 Impact of gasket thermal conductivity on the TCR for different contact materials: (a) Stainless steel, (b) Aluminum, (c) Copper

Fig.4 shows that the thermal contact resistance decreases with the increase of the gasket thermal conductivity and its decrease is significant in the case of lower thermal conductivity materials. The obtained results are in agreement with the literature (Voller et al., 2007; O'Callaghan et al., 1988).
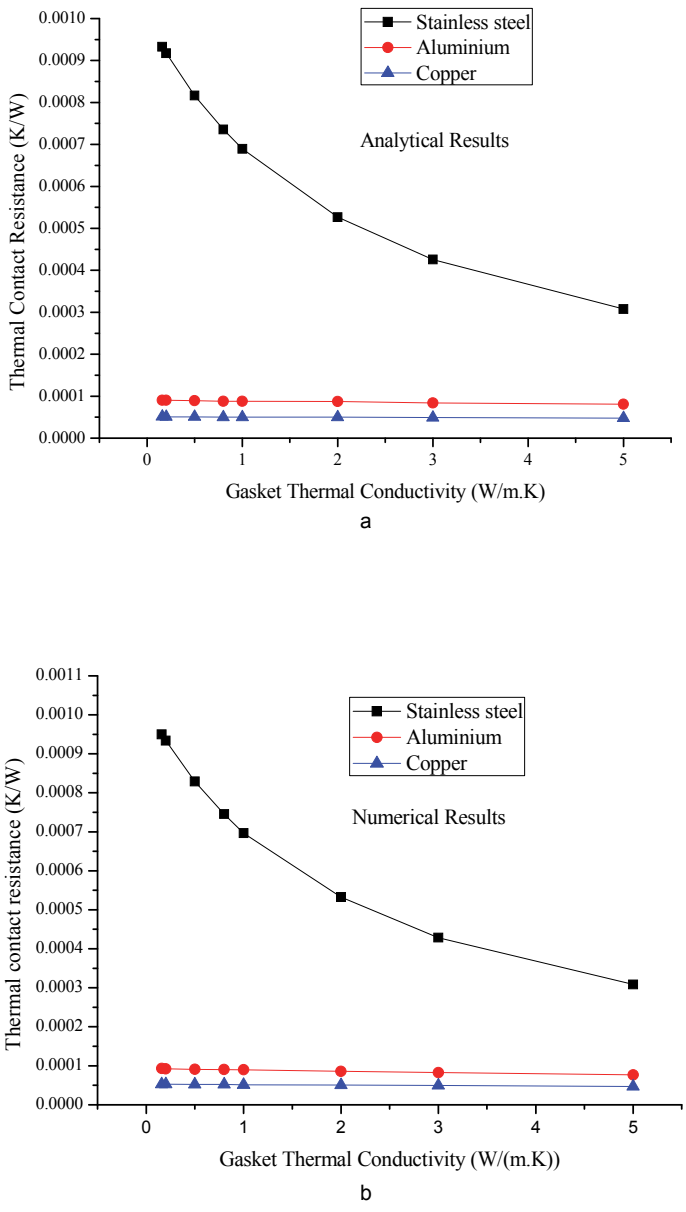

Fig. 5 Impact of gasket thermal conductivity on the TCR for different material: (a) Analytical results, (b) Numerical results

Figure 5 shows a comparison of the gasket thermal conductivity effect on the TCR for different materials in contact. It is highly remarkable that the effect of the gasket thermal conductivity decreases in the case of higher thermal conductivity materials. A good concordance is observed between analytical and numerical results.

\subsection{Decrease rate of thermal contact resistance}

Table 1 presents the rate of decrease in thermal contact resistance. The results presented in this table show that the thermal contact resistance is reduced by more than $67 \%$ in the case of stainless steel, whose thermal conductivity is $20 \mathrm{w} / \mathrm{m} \cdot \mathrm{K}$, while it is reduced by no more than $11 \%$ in the case of copper, whose thermal conductivity is 390 $\mathrm{w} / \mathrm{m} \cdot \mathrm{K}$. These results are confirmed by the analytical model, which shows a reduction of around $70 \%$ for stainless steel and around $10 \%$ for copper. These results confirm that the thermal contact resistance is more influenced by the quality of the gasket in the case of lower thermal conductivity materials.

In the literature, most of numerical and experimental studies of the gasket effects on TCR are based on the gasket thickness effect, which strongly reduce the TCR (Zhang et al., 2010; Voller et al., 2007), contrarily, there has not been any work on the gasket thermal conductivity effect on the TCR which is the subject of our study. 
Table 1 Analytical and numerical TCR decrease rate

\begin{tabular}{|c|c|c|c|c|}
\hline Material & $\begin{array}{c}\text { Thermal } \\
\text { conductivity } \\
w / m \cdot K\end{array}$ & $\begin{array}{c}\text { Numerical } \\
\text { decrease } \\
\text { rate of } \\
\text { TCR }\end{array}$ & $\begin{array}{c}\text { Analytical } \\
\text { decrease } \\
\text { rate of TCR }\end{array}$ & $\begin{array}{c}\text { Error } \\
\text { rate } \\
\text { between } \\
\text { the two } \\
\text { model }\end{array}$ \\
\hline $\begin{array}{c}\text { Stainless } \\
\text { steel }\end{array}$ & 20 & $67.56 \%$ & $69.98 \%$ & $3.58 \%$ \\
\hline Aluminum & 220 & $19.96 \%$ & $16,43 \%$ & $3.12 \%$ \\
\hline Copper & 390 & $10.21 \%$ & $10.01 \%$ & $1.95 \%$ \\
\hline
\end{tabular}

Fig. 6 presents the rate of decrease in TCR as a function of the thermal conductivity of the contact materials. It is shown that the numerical model results are close to the analytical model results.

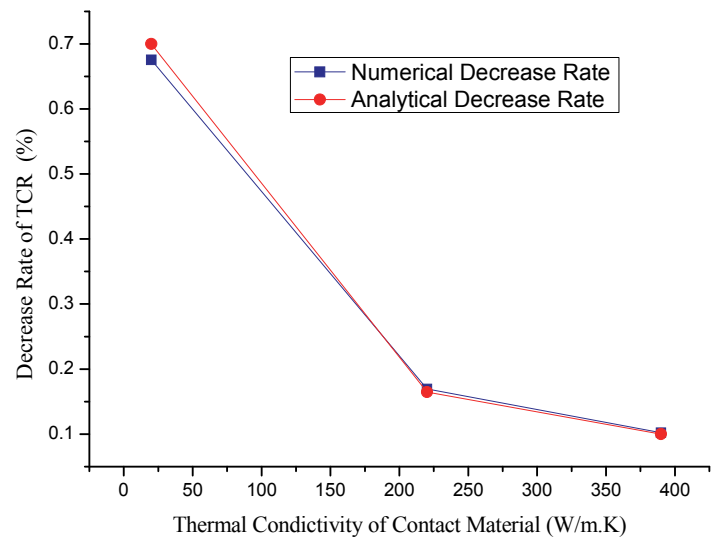

Fig. 6 TCR decrease rate as a function of contact materials thermal conductivity.

\section{CONCLUSION}

The study presented in this paper is a 2D Finite Element Method simulation of a rough contact between two materials. The aim of this study was to investigate the impact of the gasket thermal conductivity on thermal contact resistance "TCR". The TCR was computed for three pairs of materials, by varying the gasket thermal conductivity. Results obtained show that:

1- The gasket has a very important influence in heat transfer between solids in contact especially in the case of low thermal conductivity contact materials.

2- The rate of decrease in TCR decreases with increasing of contact materials thermal conductivity.

3- Numerical results and analytical results are in a very good agreement. It is necessary to use a high thermal conductivity gasket when the aim is to make a contact between two low thermal conductivity solids. It is also recommended to develop a model that takes into account the effect of the gasket thickness and its thermal conductivity on the resistance to thermal contact. Performing some experimental tests will make it possible to verify the accuracy of our results. In future work, it would be very interesting to study the gasket effect on the thermal contact resistance in three dimensions.

\section{NOMENCLATURE}

$\begin{array}{ll}\text { D } & \text { Width }(\mathrm{m}) \\ \mathrm{k} & \text { Thermal conductivity }(\mathrm{w} / \mathrm{m} \cdot \mathrm{K}) \\ \mathrm{N} & \text { Number of asperities } \\ \mathrm{q} & \text { Heat flux }\left(\mathrm{w} / \mathrm{m}^{2}\right)\end{array}$

$\begin{array}{ll}\mathrm{S} & \text { Surface }\left(\mathrm{m}^{2}\right) \\ \mathrm{Ra} & \text { Surface Roughness }(\mathrm{m}) \\ \mathrm{T} & \text { Temperature }(\mathrm{K}) \\ \Delta \mathrm{T} & \text { Jump temperature }(\mathrm{K}) \\ \mathrm{z} & \text { Coordinate }(\mathrm{m}) \\ \text { Subscripts } & \\ 1 & \text { Solid } 1 \\ 2 & \text { Solid } 2 \\ \text { ch } & \text { Hot } \\ \mathrm{f} & \text { cold }\end{array}$

\section{REFERENCES}

Belghith, S., Mezlini, S., BelhadjSalah, H., and Ligier, J.L., 2013, "Thermo-Mechanical Modeling of the Contact Between Rough Surfaces Using Homogenization Technique," Mechanics Research Communications, 53, 57-62.

http://dx.doi.org/10.1016/j.mechrescom.2013.08.004

Belghith, S., Mezlini, S., BelhadjSalah, H., Ligier, J.L., 2010, "Modeling of Contact between Rough Surfaces Using Homogenization Technique," Comptes Rendus Mécanique, 338, 48-61.

Benjamin, S., and Dominic, G., 2016, "Measurement of Thermal Interface Conductance at Variable Clamping Pressures Using a Steady State Method," Applied Thermal Engineering 96, 671-681. http://dxdoi.org/10.1016/j.appithermaleng.2015.12.010

Cames-Pintaux. A.M., and Padet, J.P., 1980, "Study of Thermal Transient Contacts- Proposal of a Thermally Equivalent Model," International Journal of Heat and Mass Transfer 23, 981-990.

Degiovanni, A., and Xiaojing Yin Zhang, 1998, "Model of Thermal Contact Resistance between Two Cylindrical Surfaces: 3D Microscopic Approach," International Journal of Heat and Mass Transfer, 41(3), 601-612.

Dongmi, B., Huanxin, Chen, and Ye, Tian., 2015, "Influence of Temperature and Contact Pressure on Thermal Contact Resistance at Interfaces at Cryogenic Temperatures," Cryogenics 52, 403-409. http://dx.doi.org/10.1016/j.cryogenics.2012.03.006

Dureja. A.K., Pawaskar, D.N., Sesbou, P., Sinha, S.K., and Sinha, R.K, 2015, "Experimental Determination of Thermal Contact Conductance Between Pressure and Calandria Tubes of Indian Pressured Heavy Water Reactors," Nuclear Engineering and Design 284, 60-66. http://dx.doi.org/10.1016/j.nucengdes.2014.11.025

Loulou. T., Abou-Khachfe, R., and Bardon, J.P., 1999, "Estimation of the Thermal Contact Resistance during Glass Solidification," International Journal of Thermal Sciences, 38(11), 984-998.

Madhusudana, C.V, "Thermal Contact Conductance," 1996, SpringerVerlag. New York.

Navni. N., Verma. and Sandip, M, 2016, "Extraction of Thermal Contact Conductance of Metal-Metal Contacts From Scale-Resolved Direct Numerical Simulation," International Journal of Heat and Mass Transfer, 94, 164-173.

http://dx.doi.org/10.1016/j.nucengdes.2015.11.026

O'Callaghan, P.W., and Probert, S.B., 1988, "Reducing the Thermal Resistance of a Pressed Contact," Applied Energy, 30(1), 53-60.

Qingyn. T., Jingjing, H., and Weifang, Z, 2015, "Influencing Factors of Thermal Contact Conductance between TC4/30CrMnSi Interfaces," International Journal of Heat and Mass Transfer, 86, 694-698. http://dx.doi.org/10.1016/j.ijheatmasstransfer.2015.03.035

Voller. G.P., and Tirovic, M., 2007, "Conductive Heat Transfer across a Bolted Automotive Joint and the Influence of Interface Conditioning," 
International Journal of Heat and Mass Transfer, 50(23-24), 48334844.

http://dx.doi.org/10.1016/j.ijheatmasstransfer.2007.03.001

Ruifeng, D., Tianran, Ge., Xunliang, L., and Zhi, W, 2016, "Effects of Contact Pressure, Interface Temperature, and Surface Roughness on the Thermal Contact Conductance Between Stainless Steel Surface Under Atmosphere Condiction," International Journal of Heat and Mass Transfer, 94, 156-163.

http://dx.doi.org/10.1016/j.ijheatmasstransfer.2015.11.069
Zhang. X.Z., Zhang, L.W., and Xing, L., 2010, "Study of Thermal Interfacial Resistance Between TC11/Glass Lubrication/K403 Joint", Experimental Thermal and Fluid Science. 34(1), 48-52.

http://dx.doi:10.1016/j.expthermflusci.2009.09.001

Zhi. Z., Li-Wen Zhang, Qin-Ke Wu., and Sen-Dong Gu., 2013, “An Experimental Investigation of Thermal Contact Conductance of Hastelloy C-276 Based on Steady State Heat Flux Method," International Communication in Heat and Mass Transfer 41, 63-67. http://dx.doi.org/10.1016/j.icheatmasstransfer.2012.11.007 